\title{
NÍVEL DE ATIVIDADE FÍSICA, POR ACELEROMETRIA, EM IDOSOS DO MUNICÍPIO DE SÃO PAULO: ESTUDO SABE
}

\author{
PHYSICAL ACTIVITY LEVEL, BY ACCELEROMETRY, IN SÃO PAULO'S ELDERLY PEOPLE: SABE STUDY
}

NIVEL DE ACTIVIDAD FISICA, POR ACELEROMETRIA, DE LÓS ANCIANOS DE LA CIUDAD

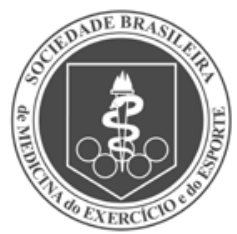

Artigo Original

ORIGINAL ARTICLE

DE SÃO PAULO: ESTUDIO SABE

Denise Rodrigues Bueno'

(Educadora Física)

Maria de Fátima Nunes Marucci ${ }^{1}$ (Nutricionista)

Manuela de Almeida Roediger ${ }^{1}$ (Nutricionista)

Igor Conterato Gomes

(Educador Físico)

Yeda Aparecida de Oliveira Duarte ${ }^{2}$ (Enfermeira)

Maria Lucia Lebrão' (Médica).

1. Universidade de São Paulo, Faculdade de Saúde Pública, São Paulo, SP, Brasil.

2. Universidade de São Paulo, Escola de Enfermagem, São Paulo,

SP, Brasil.

\section{Correspondência:}

Programa de Pós Graduação em Nutrição em Saúde Pública da Faculdade de Saúde Pública, Universidade de São Paulo Avenida Doutor Arnaldo, 715, Cerqueira César, São Paulo, SP, Brasil. 01246-904.denisebueno@usp.br

\section{RESUMO}

Introdução: Estudos populacionais utilizam, geralmente, métodos indiretos de estimativa do nível de atividade física (NAF) da população. Entretanto, o erro de medida, principalmente quando se trata da população idosa, é grande. Estudos nacionais que usam medidas objetivas são escassos. Objetivo: Este estudo buscou descrever o perfil de prática de atividade física (AF) da população idosa do município de São Paulo utilizando-se acelerometria. Métodos: Este trabalho faz parte do estudo SABE, desenvolvido na capital do Estado de São Paulo e analisou os dados de 568 idosos ( $\geq 65$ anos). O acelerômetro da marca Actigraph foi utilizado durante 3 dias consecutivos. Os idosos foram classificados segundo o NAF de acordo com as recomendações atuais de AF. O NAF foi descrito (média e IC 95\%) segundo sexo e grupos etários ( $<70$ anos, 70 a 79 anos e $\geq 80$ anos). As proporções de idosos em cada grupo de NAF foram descritas em termos relativos (\%). As diferenças entre os grupos foram estimadas utilizando-se o teste generalizado de igualdade entre médias de Wald. Resultados: Os idosos considerados sedentários representaram $63,1 \%$ (48,3\% dos homens e $71,7 \%$ das mulheres) e apresentaram média de idade maior; houve prevalência de 25,7\% dos homens e 8,13\% das mulheres classificados como fisicamente ativos, o que corresponde a apenas $14,6 \%$ do total de idosos. Os idosos gastam, em média, 12,6 (IC 95\% 10,6 - 13,7) minutos por dia em atividades físicas consideradas de intensidade moderada e/ou vigorosa. A proporção de idosos fisicamente ativos é menor quanto maior for a idade. Conclusão: O NAF da população idosa do Município de São Paulo é baixo e considerado insuficiente, principalmente para o sexo feminino. Assim, houve maior proporção de idosos considerados sedentários e insuficientemente ativos quando comparados aos ativos.

Descritores: acelerometria; idosos; atividade motora.

\section{ABSTRACT}

Introduction: Population studies generally use indirect methods to estimate the physical activity level (PAL). However, the measurement error, particularly when it comes to the elderly, is significant. National studies using objective measures are scarce. Objective: This study aimed to describe the physical activity level of the elderly in São Paulo city using accelerometry. Methods: This research is part of SABE Study carried out in the capital of São Paulo and analyzed data of 568 elderly people ( $\geq 65$ years). The Actigraph accelerometer was used for three consecutive days. The elderly were classified according to the PAL in accordance with the current recommendations of PA. The PAL was described (mean and $95 \%$ Cl) by sex and age groups ( $<70$ years, $70-79$ years and $\geq 80$ years). The proportion of elderly people in each PAL group was described in relative terms (\%). Differences between groups were estimated using Wald test. Results: Elderly classified as sedentary accounted for $63.1 \%$ (48.3\% men and $71.7 \%$ women) and had higher mean age; prevalence was $25.7 \%$ of men and $8.13 \%$ of women classified as physically active, which corresponds to only $14.6 \%$ of the elderly. The elderly spend on average 12.6 (95\% Cl 10.6 to 13.7) minutes per day in physical activity considered moderate and/ or vigorous. The proportion of elderly individuals active is smaller the greater the age. Conclusion: The PAL of elderly population of São Paulo city is low and insufficient, especially among women. Thus, the proportion of elderly people considered sedentary and insufficiently active were greater compared to physically active elderly people.

Keywords: accelerometer; elderly; motor activity.

\section{RESUMEN}

Introducción: Los estudios de población suelen utilizar métodos indirectos para estimar el nivel de actividad física (NAF) de la población. Sin embargo, el error de medición, especialmente en el caso de la población de edad avanzada, es grande. Estudios nacionales utilizando medidas objetivas son escasos. Objetivo: Este estudio trata de describir el perfil de actividad física (AF) de la población de ancianos en la ciudad de São Paulo mediante acelerometría. Métodos: Este trabajo forma parte del estudio SABE, llevado a cabo en la capital de São Paulo y analizó los datos de 568 personas de edad avanzada ( $\geq 65$ años). Se utilizó el acelerómetro Actigraph durante tres días consecutivos. Los ancianos fueron clasificados de acuerdo al NAF según las recomendaciones de AF actuales. EI NAF fue descrito (media e IC 95\%) por sexo y grupos de edad (< 70 años, 70-79 años y $\geq 80$ años). La proporción de personas mayores en cada grupo de NAF fue descrita en términos relativos (\%). Las diferencias entre los grupos se calcularon utilizando la prueba de Wald. 
Resultados: Ancianos considerados sedentarios representan el 63,1\% (48,3\% hombres y 71,7\% mujeres) y tuvieron mayor media de edad; la prevalencia fue del $25,7 \%$ de los hombres y el $8,13 \%$ de las mujeres clasificados como fisicamente activos, lo que corresponde a sólo el 14,6\% de las personas mayores. Los ancianos gastan en promedio 12,6 (IC del 95\%: 10,6 a 13,7) minutos por día en actividad física considerada moderada y/o vigorosa. La proporción de ancianos fisicamente activos disminuye a medida que aumenta la edad. Conclusión: EI NAF de la población anciana de São Paulo es considerado bajo e insuficiente, especialmente para las mujeres. Por lo tanto, hubo una mayor proporción de personas de edad avanzada considerada sedentaria e insuficientemente activa en comparación con los activos.

Descriptores: acelerometría; anciano; actividad motora..

\section{INTRODUÇÃO}

O nível de atividade física é um indicador importante de saúde da população. Em todo o mundo, a inatividade física é responsável direta por $6 \%$ dos casos de doenças coronarianas, 7\% de diabetes tipo II, 10\% de câncer de mama e por 5,3 milhões de mortes ocorridas ${ }^{1}$. Além de sua associação com a menor capacidade de mobilidade corporal $^{2}$ e maior fragilidade ${ }^{3}$, especialmente em indivíduos em idade mais avançadas.

Estima-se que 18\% dos homens e 14\% das mulheres de 65 a 74 anos atinjam à recomendação de 150 minutos de atividade física (AF) moderada na semana. Acima de 75 anos, os praticantes são apenas $8 \%$ dos homens e $4 \%$ das mulheres ${ }^{4}$. Entre os motivos observados para a menor prevalência de prática regular de AF em idosos estão as sensações associadas ao exercício, como dor no peito, desconforto, medo de cair e a preocupação com a segurança ${ }^{5}$ e a auto-percepção negativa de saúde 6 .

O nível de AF tem sido predominantemente identificado por meio de autorrelato, uma vez que se trata de um método de baixo custo e facilmente aplicável em estudos com grandes populações, em um curto período de tempo. No entanto, as desvantagens deste método limitam a leitura dos resultados com precisão, principalmente em idosos ${ }^{7-10}$.

Dadas as limitações particulares de autorrelato de AF em idosos, equipamentos de mensuração objetiva da AF, como por meio de acelerômetros, são necessários para identificar com maior precisão os níveis diários e padrões de atividade. A capacidade de avaliar, minuto a minuto, as mudanças de intensidades, sugere que se trata de um método valioso de estimativa, uma vez que há considerável variação em virtude do nível de aptidão física e relação social7. No entanto, ainda não existem estudos com acelerometria disponíveis em idosos brasileiros (> 60 anos), que descrevam padrões e níveis de AF diários em amostras representativas de grandes populações.

Além disso, com a realização de estudos representativos, com medidas objetivas de mensuração da $A F$, os resultados quanto à intensidade e volume da atividade e sua relação com doenças e incapacidades ajudaria o desenvolvimento de recomendações de AF relacionados à saúde para essa população.

Dessa forma, o objetivo deste trabalho foi analisar detalhadamente, de acordo com sexo, a distribuição e o nível de atividade física de idosos, com 65 anos de idade ou mais, do munícipio de São Paulo.

\section{MÉTODOS}

O Estudo SABE (Saúde, Bem-Estar e Envelhecimento), é um estudo epidemiológico de base domiciliar, realizado com idosos ( $\geq 60$ anos) residentes na área urbana do município de São Paulo. Os procedimentos de amostragem foram descritos por Lebrão e Duarte ${ }^{11}$.

Em 2000, foram entrevistados 2.143 indivíduos (coorte A). Em 2006, os idosos da coorte A foram entrevistados novamente e uma nova coorte probabilística de indivíduos com idade entre 60 e 64 anos foi iniciada, composta por 298 idosos (coorte B). No ano de 2010, idosos das coortes A e $B$ foram novamente entrevistados e deu-se início à coorte $C$, composta por idosos entre 60 e 64 anos. Ademais, o projeto do SABE foi aprovado pelo comitê de ética da FSP/USP e pela CONEP (Comissão Nacional de Ética em Pesquisa) pelo protocolo 475.455. Todos os idosos participantes assinaram o Termo de Consentimento Livre e Esclarecido.

Para este estudo, foi selecionada uma amostra de 568 idosos participantes do Estudo SABE, todos entrevistados na terceira etapa (2010), pertencentes às coortes A e B, que apresentavam dados de acelerometria. Portanto, trata-se de um recorte transversal do Estudo SABE, com amostra representativa da população idosa ( $\geq 65$ anos) do município de São Paulo.

Para quantificar o nível do nível de atividade física (NAF) dos idosos, foi utilizado o sensor de movimento (acelerômetro) marca Actigraph, modelo GT3X (Actigraph LLC, Pensacola, FL).

Os idosos foram orientados utilizar acelerômetro acoplado à roupa na altura da cintura, com o auxílio de uma faixa elástica, pelo período de três dias consecutivos, durante 24 horas, conforme as recomendações de Garatachea et al. ${ }^{12}$.

Para a utilização do monitor, o preparo era realizado no dia anterior à data de início do uso do equipamento. $\mathrm{O}$ aparelho era programado para iniciar a contagem a partir das $9 \mathrm{~h}$ de terça-feira e encerrar às $9 \mathrm{~h}$ de sexta-feira.

O processo download e análise dos dados registrados foi realizado pelo software Actlife versão 5.0. Foram incluídos nas análises apenas dias completos de monitoramento. Hora consecutiva de zero count (dados brutos do acelerômetro) foi considerada como o período de não utilização e dias com menos de dez horas utilizadas do aparelho foram excluídos, por aumentarem a possibilidade de maior variabilidade de dados ${ }^{12}$.

Os valores de counts foram traduzidos para minutos de AF. A intensidade da prática de AF foi analisada a partir da classificação estabelecida pela equação de Freedson et al. ${ }^{13}$ : atividade sedentária (0 - 99 contagens/minuto), leve (100 - 759 contagens/minuto), de estilo de vida (760 - 1951 contagens/minuto), moderada (1952 - 5724 contagens/ minuto), vigorosa (5725 - 9498 contagens/minuto) e muito vigorosa (> 9499 contagens/minuto).

Além do tipo de AF realizada, os idosos foram classificados em três níveis de AF: 1. Idosos Ativos (30 minutos ou mais de atividades moderadas e/ou vigorosa por dia); 2. Insuficientemente ativos (10 a 29 minutos de atividades moderadas e/ou vigorosa por dia) e 3. Fisicamente inativos ou sedentários (menos 10 minutos por dia de atividades moderadas e/ou vigorosa por dia).

Utilizaram-se as variáveis sexo (masculino e feminino) e grupos etários ( $<70$ anos, 70 a 79 anos e $\geq 80$ anos) para descrever a população de estudo, segundo os níveis de atividade física estimados pelo acelerômetro. 
Para as análises descritivas, utilizaram-se a média e o intervalo de confiança (IC95\%) para as variáveis contínuas e a proporção foi calculada para as variáveis categóricas. As diferenças entre os grupos foram estimadas utilizando-se o teste generalizado de igualdade entre médias de Wald, que levam em consideração pesos amostrais para estimativas com ponderações populacionais.

\section{RESULTADOS}

Participaram do estudo 568 idosos com média de idade de 74 anos (IC 95\%=73,4 - 74,6), sendo 63\% da amostra composta por mulheres.

Os idosos considerados sedentários representaram 63,1\% (48,3\% dos homens e $71,7 \%$ das mulheres) da amostra (Figura 1A). Os resultados apresentados na Figura 1B mostram que, segundo sexo, houve prevalência de 25,7\% dos homens e 8,13\% das mulheres classificados como fisicamente ativos, o que corresponde a apenas 14,6\% do total de idosos. Além disso, este grupo era o mais jovem, com média de idade de 70,4 (IC95\%=69,4 - 71,4) anos.

O grupo de idosos considerados sedentários foi composto pelos idosos com média de idade significativamente maior, de 75,6 (IC95\% = $74,8-76,4)$ anos. Além disso, a proporção de idosos classificados como fisicamente ativos foi menor, quanto maior o grupo etário $(p<0,001)$. Em idosos com menos de 70 anos, 22\% foram classificados como ativos, enquanto essa proporção foi menor (15\%) para os idosos com idade entre 70 e 79 anos e, em idosos com 80 anos ou mais (2\%) (Figura 1C).

Os idosos despenderam, em média, 12,6 (IC95\% 10,6 - 13,7) minutos por dia em atividades físicas consideradas de intensidade moderada e/ou vigorosa (MVPA). Os homens apresentaram valores acima da média (17,9 minutos/dia; IC95\% 14,8 - 21,1), com diferença estatisticamente significante, em relação às mulheres (8,8 minutos/ dia; IC95\% 7,4 - 10,1) (Tabela 1).
Observaram-se menores médias de MVPA em grupos etários mais velhos ( $\geq 80$ anos), para todos os idosos e segundo sexo. Os homens apresentaram maiores médias de MVPA, do que as mulheres em todos os grupos etários estudados (Tabela 1).

Para o tempo de atividades consideradas sedentárias, os idosos despendem, em média, 818 (IC95\%- 807,79 - 828,27) minutos por dia neste tipo de atividade. Esses valores também são maiores para o sexo masculino e para os grupos etários mais velhos. Entretanto, para as AF consideradas leves, as médias de tempo por dia foram semelhantes entre os sexos e menores, quanto maior a idade dos idosos.

Segundo a contagem total de AF (Vector Magnitude Counts), a AF diária de idosos com menos de 70 anos foi 13,5\% maior que a AF de idosos com idade entre 70 e 79 anos e, quase o dobro da AF diária dos idosos com 80 anos e mais. Situação semelhante quando analisada segundo sexo. Embora haja diferença entre os sexos quando a AF é analisada, segundo a intensidade, para a contagem total diária, os valores foram semelhantes.

Analisando atividades de intensidade moderadas e vigorosas separadamente, a média de AF moderada dos idosos variou de 47,9 minutos (IC95\% - 39,6 - 56,2) nos idosos mais jovens, a 10,6 minutos (IC95\%- 8,0 - 13,2) nos idosos mais velhos ( $\geq 80$ anos). Além disso, foi menor quanto maior a idade. Idosos com 80 anos e mais apresentaram valor médio menor que 1/4 da média dos idosos mais jovens. Para os homens, não houve diferença entre idosos com menos de 70 anos e idosos com até 79 anos de idade. Porém, o tempo dispendido em AF desta intensidade nos idosos mais velhos foi significativamente inferior (Tabela 2).

A realização de atividade física vigorosa ou muito vigorosa foi muito próxima a zero. Nenhum dos grupos etários chegou a realizar, em média, ao menos um minuto por dia de atividade física com esta intensidade (Tabela 2).

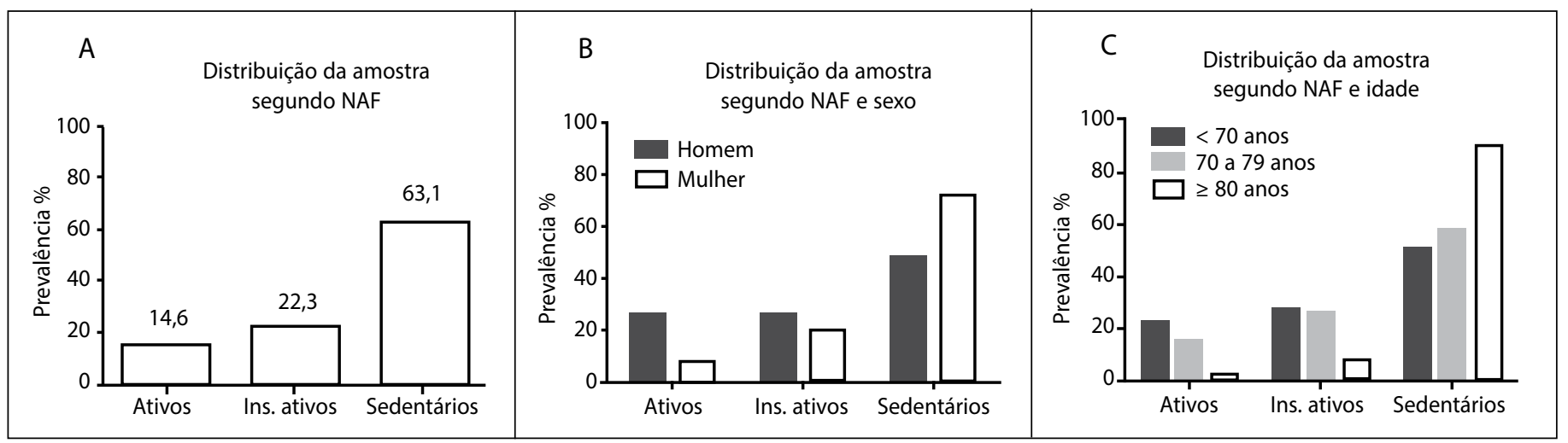

Figura 1. Distribuição (\%) de idosos, segundo nível de atividade física da amostra total (A) segundo sexo (B) e segundo grupos etários.

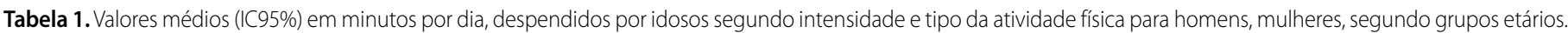

\begin{tabular}{|c|c|c|c|c|}
\hline \multirow[b]{2}{*}{ Variável } & \multicolumn{3}{|c|}{ Grupos etários } & \multirow[b]{2}{*}{$\begin{array}{c}\text { Geral } \\
\text { Média (IC95\%) }\end{array}$} \\
\hline & $\begin{array}{c}<70 \text { anos } \\
\text { Média (IC 95\%) }\end{array}$ & $\begin{array}{c}70 \text { a } 79 \text { anos } \\
\text { Média (IC 95\%) }\end{array}$ & $\begin{array}{c}\geq 80 \text { anos } \\
\text { Média (IC } 95 \%)\end{array}$ & \\
\hline \multicolumn{5}{|c|}{ Amostra total } \\
\hline Counts & $452840,5(423770,9-481910,0)$ & $391693,5(368970,4-414416,6)$ & $255040,2(234500,3-275580,1)$ & $382344,4(66610,4-398078,4)$ \\
\hline MVPA & $16,3(13,45-19,2)$ & $13,2(10,7-15,6)$ & $3,7(2,7-4,6)$ & $12,16(10,65-13,7)$ \\
\hline Sedentária & $810,1(791,2-828,8)$ & $811,4(795,7-827,2)$ & $843,8(826,2-861,4)$ & $818,03(807,79-828,27)$ \\
\hline \multicolumn{5}{|c|}{ Homens } \\
\hline Counts & $452764,6(400038,5-505490,7)$ & $392089,6(348075,4-436103,9)$ & $258938,2(219936,9-297939,4)$ & $387916.9(358559,4-417274,4)$ \\
\hline MVPA & $21,0(15,6-26,3)$ & $20,1(15,4-25,9)$ & $5,7(3,6-7,8)$ & $17,91(14,77-21,0)$ \\
\hline Sedentária & $823,9(791,7-856,1)$ & $818,4(788,7-848,0)$ & $852,1(821,79-882,4)$ & $826,75(808,23-845,3)$ \\
\hline \multicolumn{5}{|c|}{ Mulheres } \\
\hline Counts & $452888.5(418886,1-486890,9)$ & $391451.3(366413,3-416489,2)$ & $253170.6(229105,5-277235,6)$ & $379070,1(361021.8-397118,4)$ \\
\hline MVPA & $13,4(10,3-16,4)$ & $8,6(6,73-10,5)$ & $2,7(1,8-3,6)$ & $8,78(7,40-10,2)$ \\
\hline Sedentária & $801,3(778,5-824,1)$ & $807,2(789,4-825,0)$ & $839,8(818,1-861,5)$ & $812,91(800,88-824,9)$ \\
\hline
\end{tabular}


Os resultados apresentados na figura 2A-C ilustram as diferenças das contagens de AF leve, moderada e vigorosa separadamente, segundo sexo e grupos etários. Como esperado, a AF leve tem a maior média de tempo por dia, variando de 765 minutos (IC95\%- 723,2 - 806,9) em idosos com menos de 70 anos a 503 minutos (IC95\% - 467,8 - 538,2) em idosos com 80 anos ou mais. Os homens apresentaram valores abaixo da média da amostra em todos os grupos etários e, para ambos os sexos, as médias foram menores nos grupos etários mais velhos.

\section{DISCUSSÃO}

De acordo com a literatura, este é o primeiro estudo populacional de base domiciliar que analisou o NAF por meio de acelerometria em idosos residentes em São Paulo. Os resultados mostraram que a maior proporção dos idosos se apresenta no menor nível de atividade física e abaixo dos níveis recomendados para a manutenção da saúde desta população ${ }^{14}$. Verificou-se que o nível de atividade física é menor quanto maior a idade dos idosos, especialmente em mulheres, com médias menores que a observada para a população no geral. Mesmo a quantidade de atividades físicas de intensidade leve são baixas, para os idosos de idades mais avançadas.

Parte dos nossos resultados difere dos encontrados por outros estudos que utilizaram métodos semelhantes de análise, segundo os materiais utilizados e critérios de classificação. Em idosas suecas, 23\% da amostra foram classificadas como fisicamente ativas, enquanto $46 \%$ ( $\geq 65$ anos) não praticavam 30 minutos por dia de AF moderada e

Tabela 2. Valores médios (IC95\%) em minutos por dia, despendidos por idosos segundo intensidade e tipo da atividade física para homens, mulheres, segundo grupos etários.

\begin{tabular}{|c|c|c|c|}
\hline & \multicolumn{3}{|c|}{ Grupos Etários } \\
\hline & $\begin{array}{c}<70 \text { anos } \\
\text { Média minutos/dia } \\
\text { (IC } 95 \%)\end{array}$ & $\begin{array}{c}70 \text { a } 79 \text { anos } \\
\text { Média minutos/dia } \\
\text { (IC } 95 \%)\end{array}$ & $\begin{array}{c}\quad \geq 80 \text { anos } \\
\text { Média minutos/dia } \\
\text { (IC } 95 \%)\end{array}$ \\
\hline \multicolumn{4}{|c|}{ Amostra total } \\
\hline Leve & $765,0(723,2-806,9)$ & $706,42(673,0-739,8)$ & $503,0(467,8-538,2)$ \\
\hline Moderada & $47,9(39,6-56,2)$ & $39,2(31,9-46,5)$ & $10,6(8,0-13,2)$ \\
\hline Vigorosa & $0,42(-0,06-0,9)$ & $0,18(-0,10-0,46)$ & $0,02(-0,01-0,05)$ \\
\hline Muito Vigorosa & $0,24(-0,23-0,7)$ & $0,01(-0,01-0,02)$ & $00,00(---)$ \\
\hline \multicolumn{4}{|c|}{ Homens } \\
\hline Leve & $733,0(663,6-802,4)$ & $662,4(608,5-716,3)$ & $485,9(429,2-542,5)$ \\
\hline Moderada & $60,8(45,6-75,9)$ & $61,4(45,5-77,2)$ & $16,1(10,3-21,8)$ \\
\hline Vigorosa & $0,96(-0,29-2,20)$ & $0,05(-0,05-0,14)$ & $0,00(--)$ \\
\hline Muito Vigorosa & $0,62(-0,60-1,83)$ & $0,00(---)$ & $0,00(---)$ \\
\hline \multicolumn{4}{|c|}{ Mulheres } \\
\hline Leve & $785,3(733,2-837,4)$ & $733,3(691,3-775,4)$ & $511,2(466,6-555,8)$ \\
\hline Moderada & $39,7(30,5-49,0)$ & $25,6(20,0-31,2)$ & $7,9(5,3-10,5)$ \\
\hline Vigorosa & $0,08(0,02-0,14)$ & $0,26(-0,18-0,71)$ & $0,03(-0,01-0,07)$ \\
\hline Muito Vigorosa & $0,00(---)$ & $0,01(-0,01-0,03)$ & $0,00(---)$ \\
\hline
\end{tabular}

vigorosa $^{15}$. Do mesmo modo, a prevalência de idosas fisicamente ativas ( $\geq 80$ anos) na Suíça ${ }^{8}$ foi maior que a observada no presente estudo (14\% vs 2\%), assim como a média de minutos por dia em MVPA (13 minutos vs 2,7minutos). Por outro lado, os resultados apresentados por Harris et al. ${ }^{16}$ mostraram prevalência maior $(89,5 \%)$ de sedentarismo e menor (2,5\%) de praticantes de AF moderada $\geq 30 \mathrm{~min} /$ dia. Gao e Tsang ${ }^{17}$ também observaram média de idade maior, em idosos classificados como sedentários ( $p<0,01)$.

Neste estudo, a média de tempo despendido em AF moderada e vigorosa é maior em homens, independente do grupo etário. E, considerando a atividade física de intensidade leve, o grupo das mulheres apresentou maior média. Comparado a outros estudos, este mesmo resultado é observado ${ }^{7}$. Os resultados de estudos realizados com estimativas de AF a partir de questionários mostraram que os homens têm preferência por atividades esportivas e competitivas ${ }^{18}$ e, mulheres, despendem mais tempo em atividades domésticas ${ }^{19,20}$. Este padrão pode explicar o maior nível de atividade física moderada em vigorosa do sexo masculino, uma vez que a prática esportiva requer maior gasto energético e intensidade de esforço.

Considerando o sexo masculino, um estudo francês com idosos de idade $\geq 70$ anos verificou resultados semelhantes aos nossos, onde a média de MVPA diária foi de 23,8 minutos por dia, apenas pouco mais de 3 minutos por dia superior aos resultados do presente estudo ${ }^{7}$.

Estes valores são inferiores aos observados na população mais jovem, dos indivíduos com menos de 60 anos no Brasil e com menos de 65 anos nos países desenvolvidos ${ }^{7,17}$. Uma possível explicação para isso pode ser a preferência entre os jovens e adultos por atividades esportivas, que em geral, são atividades mais intensas quando comparadas às atividades praticadas por idosos, como a caminhada ${ }^{21}$. Além disso, a participação em atividades físicas depende de uma interação complexa entre variáveis sociais, ambientais, psicológicas e físicas ${ }^{22}$.

O delineamento transversal do estudo não permite afirmar se a menor capacidade funcional, presente no processo de envelhecimento, foi uma variável contribuinte para o menor NAF nos indivíduos com idades mais avançadas. Porém, não foi objetivo deste estudo analisar quais seriam as variáveis associadas à inatividade física em idosos. Entretanto, estudos têm mostrado que a redução do nível de atividade física com o envelhecimento pode ser influenciada pela diminuição de capacidade funcional motora, devido, principalmente, por mudanças da composição corporal, especialmente pela redução do componente de massa muscular ${ }^{23}$

Por outro lado, além das variáveis biologias, variáveis sociais podem interferir no NAF de idosos. Como observado por Bueno et al. ${ }^{24}$, que observaram que após os 60 anos de idade há redução do NAF no domínio ocupacional, inferindo que o tempo dedicado ao trabalho é uma variável importante para determinar o NAF de idosos e que, o tempo disponível ocioso após a aposentadoria não foi preenchido com a prática de AF de lazer.

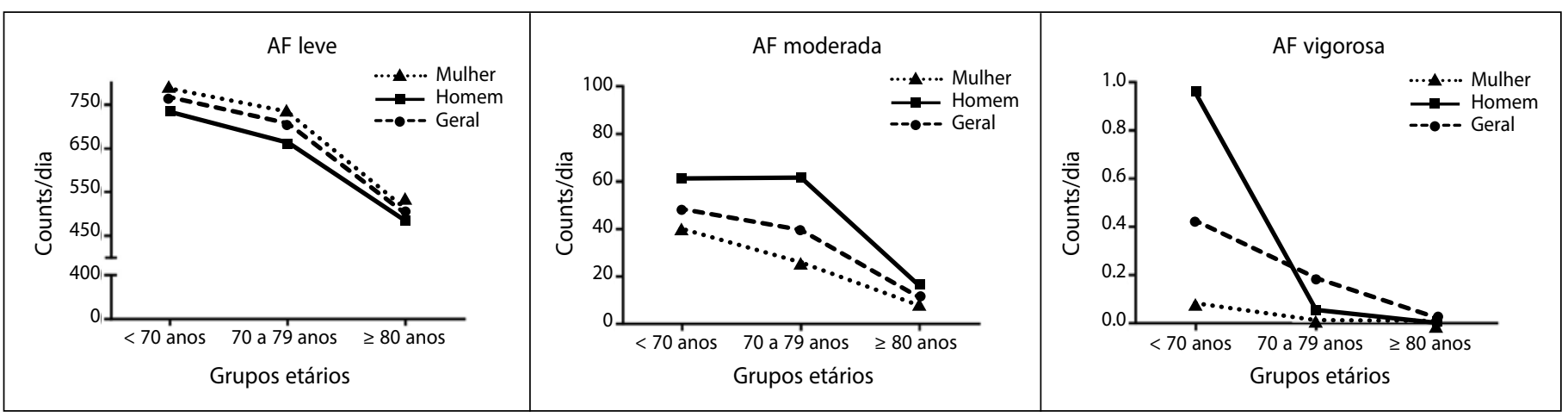

Figura 2. Contagens por dia de atividade física Leve (A), moderada (B) e vigorosa (C), segundo sexo e grupos etários. 
Assim, pode-se inferir que mais esforços devem ser direcionados no intuito de promover a prática da AF nesta população, uma vez que o estilo de vida fisicamente ativo contribui para o menor risco de desenvolvimento de doenças crônicas, como hipertensão arterial e diabete melito tipo 2. Doenças cuja prevalência é crescente na população idosa e as estratégias clínicas de controle são onerosas ao sistema de saúde ${ }^{25,26}$. As recomendações internacionais sugerem o mínimo de 30 minutos por dia dispendidos em AF de intensidade moderada ou vigorosa para a manutenção da saúde ${ }^{14,27}$. Como observado nos resultados deste estudo, a maioria da população idosa não atinge à recomendação.

Vale ressaltar que, considerando o aumento da população de idosos no Brasil, estima-se que os custos com previdência (aposentadorias e pensões) dobrarão até $2050^{28}$. Assim, a estimativa de custos, levando em consideração a especulação de maior prevalência de doenças crônicas, causa preocupação quanto ao impacto econômico às despesas governamentais com saúde nas próximas décadas. Nesse sentido, estudos enfatizam que a estratégia de prevenção primária, por meio da inclusão da AF no estilo de vida, pode ser melhor em termos te custo efetividade, quando comparada ao uso de medicamentos ${ }^{29}$.

Entre as limitações do estudo, a utilização do acelerômetro por três dias deve ser considerada, por dificultar a classificação do NAF em fisicamente ativos ou insuficientemente ativos. Porém, Hart et al. ${ }^{30}$ argumentam que para estimar o nível de atividade física em idosos, considerando as intensidades moderada e vigorosa, é justificável o uso do monitor de acelerometria em apenas dois dias, como forma de viabilizar a utilização do acelerômetro em estudos populacionais empregados em grandes metrópoles, como a cidade de São Paulo, no Brasil.

Como mencionado anteriormente, o perfil inédito deste estudo é uma característica relevante a ser citada, uma vez que o estudo foi conduzido com uma amostra representativa da população idosa do município de São Paulo, a maior cidade brasileira em número de habitantes. Por fim a alta correlação observada ( $r=0,83)$, entre este tipo de medida com métodos considerados padrão outro para a estimativa de $N A F^{31}$, como a água duplamente marcada, contribui consideravelmente para a confiabilidade dos resultados encontrados.

\section{CONCLUSÃO}

O nível de atividade física da população idosa do Município de São Paulo é considerado insuficiente, segundo as recomendações internacionais de AF para esta população, especialmente tratando-se do sexo feminino. Assim, na população idosa paulistana, houve maior proporção de idosos considerados sedentários e insuficientemente ativos, que aumentou quanto maior a idade dos indivíduos analisados.

Todos os autores declararam não haver qualquer potencial conflito de interesses referente a este artigo.

CONTRIBUIÇÕES DOS AUTORES: DRB (0000-0002-9022-5291)*, ICG e MAR foram os principais contribuintes na análise estatística e redação do manuscrito. MFNM (0000-0002-2908-7280)*, YAOD (0000-0003-3933-2179)* e MLL contribuíram com a revisão do manuscrito e com o conceito intelectual do estudo. Todos os autores aprovaram a versão final do trabalho. *Número ORCID (Open Researcher and Contributor ID).

\section{REFERÊNCIAS}

1. Lee IM, Shiroma EJ, Lobelo F, Puska P, Blair SN, Katzmarzyk PT, et al. Effect of physical inactivity on major non-comunicable diseases worldwide: an analysis of burden of disease and life expectancy. Lancet. 2012;380(9838):219-29.

2. Santos VR, Gomes IC, Santos LL, Agostinete RR, Freitas Júnior IF. Associação entre fatores de risco cardiovascular e capacidade funcional de idosos longevos. Medicina (Ribeirão Preto). 2013;46(1):10-6.

3. Costa TB, Neri AL. Medidas de atividade física e fragilidade em idosos: dados do FIBRA Campinas, São Paulo, Brasil. Cad Saúde Pública. 2011;27(8):1537-50.

4. National Centre for Social Research. Health Survey for England 2004. London: Department of Health; 2005.

5. Resnick B, Spellbring AM. Understanding what motivates older adults to exercise. J Gerontol Nurs. 2000;26(1):34-42.

6. Mathews AE, Laditka SB, Laditka JN, Wilcox S, Corwin SJ, Liu R, et al. Older adults' perceived physical activity enablers and barriers: a multicultural perspective. J Aging Phys Activ. 2010;18(2):119-40

7. Davis MG, Fox KR. Physical activity patterns assessed by accelerometry in older people. Eur J App Physiol. 2007;100(5):581-9.

8. Gerdhem P, Dencker M, Ringsberg K, Akesson K. Accelerometer-measured daily physical activity among octogenerians: results and associations to other indices of physical performance and bone density. Eur J Appl Physiol. 2008;102(2):173-80

9. Murphy SL. Review of physical activity measurement using accelerometers in older adults: Considerations for research design and conduct. Prev Med. 2009;48(2):108-14.

10. Arnardottir NY, Koster A, Domelen DRV, Brychta RJ, Caserotti P, Eiriksdottir G. Objective measurements of daily physical activity patterns and sedentary behavior in older adults: Age, Gene/Environment Susceptibility-Reykjavik Study. Age and Ageing. 2013;42(2):222-9.

11. Lebrão, ML, Duarte, YAO. O projeto SABE no município de São Paulo: uma abordagem inicial. Organização Pan-Americana de Saúde: Brasília; 2003.

12. Garatachea N, Luque GT, Gallego JG. Physical activity and energy expenditure measurements using accelerometers in older adults. Nutr Hosp. 2010;25(2):224-30.

13. Freedson PS, Melanson E, Sirard J. Calibration of the Computer Science and Applications, Inc. accelerometer. Med Sci Sports Exerc. 1998;30(5):777-81

14. World Health Organization. Global recommendations on physical activity for health, 2010 [acessado em 2014/04/25]. Disponível em: http://www.who.int/dietphysicalactivity/factsheet_recommendations/en/index.html.

15. Orsini N, Bellocco R, Bottai M, Hagströmer, Sjöström M, Pagano M, et al. Profile of physical activity behaviors among Swedish women aged 56-75 years. Scand J Med Sci Sports. 2008;18(1):95-101.

16. Harris TJ, Owen CG, Victor CR, Adams R, Cook DG. What factors are associated with physical activity in older people, assessed objectively by accelerometry? Br J Sports Med. 2009;43(6):442-50.

17. Gao KL, Tsang WW. Use of accelerometry to quantify the physical activity level of the elderly. Hong Kong Physiother J. 2008;26(1):18-23.

18. Deaner RO, Geary DC, Puts DA, Ham SA, Kruger J, Fles E, et al. A sex difference in the predisposition for physical competition: males play sports much more than females even in the contemporary U.S. PLoS One. 2012;7(11):e49168

19. Haley C. Andel R. Correlates of physical activity participation in community-dwelling older adults. J Aging Phys Act. 2010;18(4):375-89

20. Moschny A, Platen P, Klaassen-Mielke R, Trampisch U, Hinrichs T. Physical activity patterns in older men and women in Germany: a cross-sectional study. BMC Public Health. 2011;11:559.

21. Salvador EP, Florindo AA, Reis RS, Costa EF. Percepção do ambiente e prática de atividade física no lazer entre idosos. Rev Saúde Pública. 2009;43(6):972-80.

22. Van Stralen MM, De Vries H, Mudde A, Bolman C, Lechner L. Determinants of initiation and maintenance of physical activity among older adults: a literature review. Health Psychol Rev. 2009;3:147-207.

23. Orsatti FL, Dalanesi RC, Maesta N, Nahas EAP, Burini RC. Muscle strength reduction is related to muscle loss in women over the age of 40. Rev Bras Cineantropom Desempenho Hum. 2011;13(1):36-42.

24. Bueno DR, Rosa CSC, Codogno JS, Freitas Junior IF, Monteiro HL. Nível de atividade física, comorbidades e idade de pacientes hipertensos. Motriz, Rio Claro. 2013;19(3);S16-24

25. Pardell H, Tresserras R, Armario P, Del Rey RH. Pharmacoeconomic considerations in the management of hypertension. Drugs. 2000;59(Suppl 2):13-20.

26. American Diabetes Association. Economic costs of diabetes in the U.S. in 2007. Diabetes Care 2008;31(3):596-615.

27. Nelson ME, Rejeski WJ, Blair SN, Duncan PW, Judge JO, King AC, et al. Physical activity and public health in older adults: recommendation from the American College of Sports Medicine and the American Heart Association. Circulation. 2007:116(9):1094-105.

28. Gragno-Lati M, Jorgensen OH, Rocha R, Fruttero A. Envelhecendo em um Brasil mais Velho: Implicações do envelhecimento populacional sobre crescimento econômico, redução da pobreza, finanças públicas e a prestação de serviços. Washington: Banco Mundial LAC; 2011

29. Li R, Zhang P, Barker LE, Chowdhury FM, Zhang X. Cost effectiveness of interventions to prevent and control diabetes mellitus: a systematic review. Diabetes Care. 2010;33(8):1872-94

30. Hart TL, Swartz AM, Cashin SE, Strath SJ. How many days of monitoring predict physical activity and sedentary behaviour in older adults? Int J Behav Nutr Phys Act. 2011;8:62-8.

31. Gardner AW, Poehlman ET. Assessment of free-living daily physical activity in older claudicants: validation against the doubly labeled water technique. J Gerontol A Biol Sci Med Sci. 1998;53(4):M275-80 